
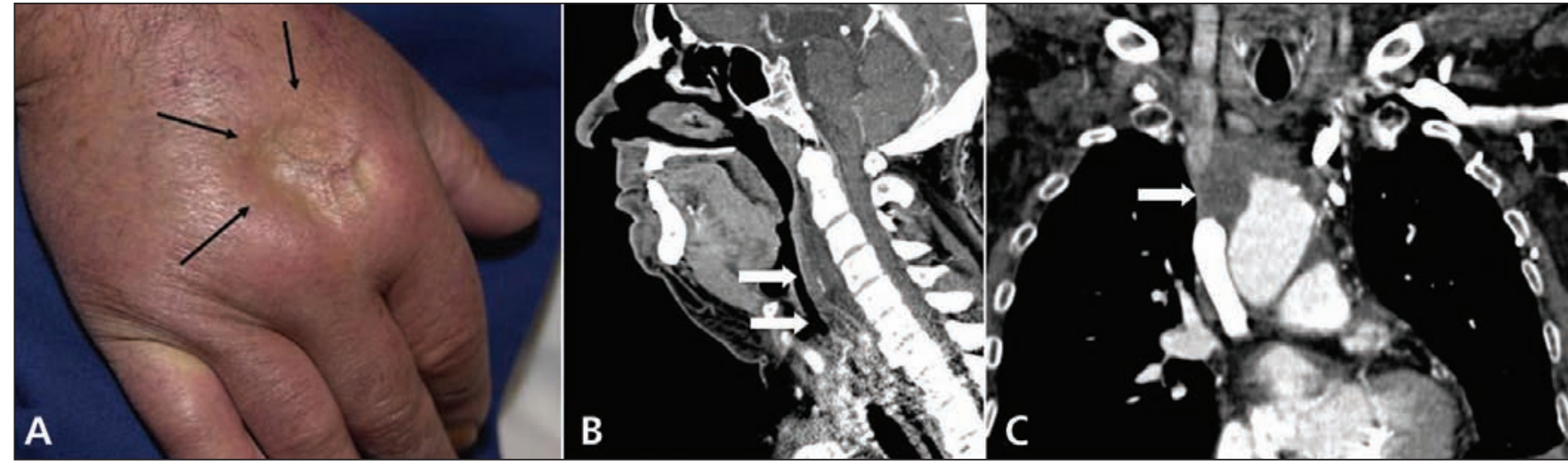

Figure 1: (A) Swelling in the hand of a 72-year-old man with signs of pitting edema ( $A$, arrows). Contrast-enhanced computed tomography scans of the neck and chest showing enlargement of the retropharyngeal space ( $B$, arrows) and a mass compressing the superior vena cava (C, arrow).

\title{
Superior vena cava syndrome
}

$\Lambda$ 72-year-old man who was overweight and had a history of hypertension and heavy smoking presented with a swollen neck. He had no dysphagia. He reported that he had experienced increased snoring and daytime sleepiness during the previous 3 weeks. An otolaryngologic examination was unremarkable except for an unusually narrow upper airway. On inspection, the patient's hands were swollen and showed signs of pitting edema (Figure 1A). Inflammatory parameters and complete blood count were normal. Contrast-enhanced computed tomography scans of the neck and chest revealed an enlarged retropharyngeal space (Figure 1B) and a mass compressing the superior vena cava (Figure 1C). A transthoracic needle biopsy showed a non-small-cell carcinoma. Radiotherapy resulted in clinical improvement and a rapid reduction in compression of the superior vena cava.

Although the exact incidence of superior vena cava syndrome remains unknown, the condition affects about 15000 patients in the United States each year. ${ }^{1}$ It has mainly malignant causes. Nonmalignant causes can include aneurysm of the aorta, thromboses after implanted intravascular catheters or fibrosing mediastinitis. Classically, compression of the superior vena cava leads to visible swelling and venous distension in the face, neck, chest and upper limbs. Other symptoms of varying severity can occur, from cough, hoarseness and dyspnea to headache, confusion and visual symptoms. In patients who are overweight, swelling of the neck may go unnoticed, and signs such as obstructive sleep apnea or edema of the upper limb may point to the diagnosis.
Clinical images are chosen because they are particularly intriguing, classic or dramatic.

Submissions of clear, appropriately labelled high-resolution images must be accompanied by a figure caption and the patient's written consent for publication. A brief explanation (300 words maximum) of the educational significance of the images with minimal references is required.
Basile Nicolas Landis MD

Department of Otolaryngology,

Head and Neck Surgery

Pierre Bohanes MD

Department of Oncology

Romain Kohler MD

Department of Radiology

University of Geneva Medical School

Geneva, Switzerland

This article has been peer reviewed.

Competing interests: None declared.

\section{REFERENCE}

1. Wilson LD, Detterbeck FC, Yahalom J. Clinical practice. Superior vena cava syndrome with malignant causes. $N$ Engl J Med 2007;356:1862-9. 